\title{
Magnetic helicity evolution inside a hexagonal convective cell
}

\author{
Aimilia Smyrli ${ }^{1,2}$, Duncan Mackay ${ }^{2}$ and Francesca Zuccarello ${ }^{1}$ \\ ${ }^{1}$ Dipartimento di Fisica e Astronomia - Sezione Astrofisica, \\ Via S. Sofia 78, 95123 Catania, Italy \\ email: emilia@oact.inaf.it \\ ${ }^{2}$ School of Mathematics and Statistics, University of St. Andrews, \\ The North Haugh, St. Andrews, \\ Fife KY 169SS, Scotland, U.K.
}

\begin{abstract}
Magnetic helicity has received considerable attention in the area of fluid dynamics. Recently, this quantity is attracting the interest of solar physicists and much research has been carried out related to magnetic helicity generation and transport through different solar layers, starting from the interior and the convection zone, towards the photosphere, the corona and finally into the heliosphere. Taking into account the global importance of supergranular cells in convection theories, we study the motion of magnetic features into such a geometrical element simplified as hexagonal cell and we analyse the results in terms of the accumulated magnetic helicity. We compute the emergence of a bipole inside the hexagonal cell and its motion from the centre of the cell towards its sides and its vertices, where the magnetic elements are considered to be sinking down. Multiple bipoles are also considered and phenomena such as cancellation, coalescence and fragmentation are also investigated. We find that the most important process for the accumulation of magnetic helicity is the shear motion between the polarities. The magnetic helicity accumulation changes its trend when one polarity reaches the side of the hexagon, and later the vertex. It has zero value when there is no shear motion inside the hexagonal cell, and it is constant when there is no shear between the two polarities during their motion along the cell sides.
\end{abstract}

Keywords. Sun: photosphere, Sun: interior, convection, Sun: magnetic fields

\section{Introduction}

The study of magnetic helicity is an important topic in solar physics, as it can describe the evolution of a magnetic field showing its complex geometry and mixing properties (such as the twist, linkage and braiding of the magnetic field lines Berger \& Field 1986).

Observationally, the study of magnetic helicity can be applied to solar eruptive phenomena such as flares (Park 2010), filaments (Mackay \& Gaizuaskas 2003; Romano et al. 2003) and coronal mass ejections (Smyrli et al. 2010). We can obtain information on the topological properties of the magnetic field from the study of magnetic helicity. It is important to investigate the build-up of this quantity in the photospheric layer of both the large and the small scale field. Here we only consider the small scale field so-called magnetic carpet configuration and in a first attempt only a small portion of it, i.e inside a single supergranular cell.

The aim of this work is to study the magnetic helicity behaviour in processes occurring inside convective (hexagonal) cells that are the result of energy transport through convection from the solar interior and lead to the evolution of the magnetic carpet and consequently drive coronal interactions (Priest et al. 2002) and rearrangements in the outer solar atmosphere. 
In particular, we study the following processes related to the motions of magnetic features :

- flux emergence (two magnetic features of opposite but equal amounts of flux appearing simultaneously).

- fly-by or advection (two polarities are moving relative to one another without any interaction between them).

- flux cancellation (two magnetic concentrations of opposite sign disappearing while approaching and interacting each other).

- coalescence (two adjacent magnetic features of the same polarity moving towards each other and merging into a larger polarity).

- fragmentation (a large magnetic concentration splitting into two smaller polarities of equal flux)

\section{The Simulation}

In order to investigate the influence of different motions of magnetic features on magnetic helicity evolution, we perform a $2-\mathrm{D}$ simulation in cartesian coordinates. The computational domain is a square box of size $256 \times 256$ pixels $\left(24000 \times 24000 \mathrm{~km}^{2}\right)$, and the resolution is 0.129 arcsec/pixel. We represent the supergranular cell with an idealized hexagon of length $L=7200 \mathrm{~km}$, in accordance with the typical values of the diameter of a supergranular cell (Parnell 2001, Mackay et al. 2002) and each magnetic element by a circle of radius $r_{0}=0.10 \mathrm{~L}$. We assume that each feature is characterized by a magnetic field with a Gaussian profile and peak strength of $100 \mathrm{G}$.

Initially we study the case of a single bipole emerging at a random position inside a box, located at the centre of the supergranular cell, having a length $\mathrm{l}=\mathrm{L} / 2$. Each magnetic polarity moves radially inside the hexagonal cell and as soon as it reaches the edge it moves towards the nearest vertex, which represents a stagnation point, and here it stops. We consider that the flow towards and along the cell boundaries is uniform with a constant speed for each polarity equal to $0.5 \mathrm{~km} / \mathrm{sec}$. The computational run has 800 time steps and each step corresponds to $0.5 \mathrm{~min}$. We consider all the possible relative motions for a single bipole by dividing the hexagonal cell into six equilateral triangles and keeping one polarity (positive) inside one triangle while allowing the other (negative) to move in each one of the six triangles. In a second step, we consider the multiple bipole case, where new bipoles emerge simultaneously in the same manner as described above. For this case we can study different processes (such as the magnetic flux cancellation and coalescence) for different numbers of bipoles (maximum 5). For each computational case we calculate the accumulated amount of magnetic helicity using the method developed by Chae (2001).

\section{Discussion and Conclusions}

- Single bipole. The fly-by case gives a larger amount of accumulated helicity (for total, positive and negative concentrations) compared to the cancellation, emergence, fragmentation or coalescence cases. When the vertical distance between the two polarities is smallest (a condition that in our simulation means strongest shear between the two magnetic polarities), the enhancement of magnetic helicity accumulation reaches a maximum (Fig. 1, top row).

- Multiple bipoles. When the number of emerging bipoles increases, the amount of accumulated helicity at the end of the computational time increases. The rate of change of magnetic helicity, shows some spikes when the number of bipoles is greater than one 

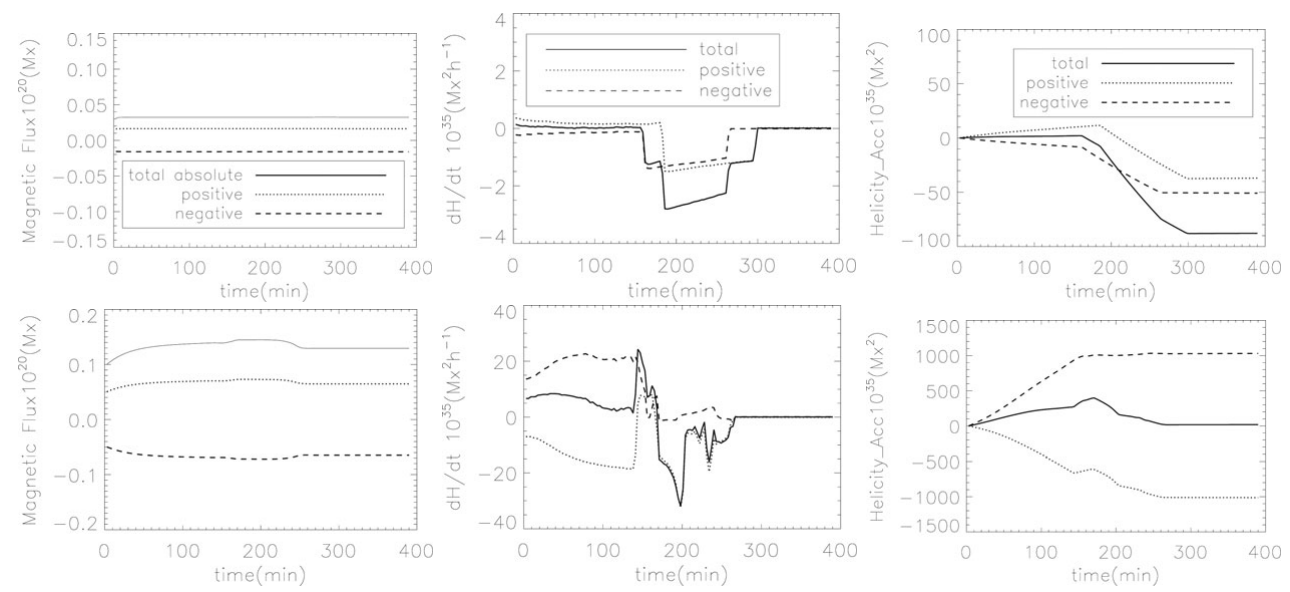

Figure 1. Top row: the bipole fly-by case, (from left to right) magnetic flux, magnetic helicity rate and magnetic helicity accumulation as a function of time. Second row: the 5-bipole case.

and the total magnetic flux shows an abrupt change. The magnetic helicity accumulation trend for positive and negative concentrations is similar for all the cases of bipoles until they reach the border of the hexagon; later on the behavior may change, depending on each case. The total accumulated amount of helicity shows sudden changes (from positive to negative inclination and reverse) almost at the same time as changes in the total magnetic flux occur (Fig. 1, bottom row). Moreover, this study shows that fragmentation is the process that mainly affects the helicity evolution.

We have studied the 2D motion of magnetic concentrations inside a hexagonal cell. This is a first attempt to interpret the behaviour of magnetic helicity for the solar magnetic carpet configuration which can later be used as a tool for interpreting helicity in higher levels of the solar atmosphere for eruptive events. The results show that the shearing motions of flux elements on the borders of the hexagonal cell provokes a large amount of helicity to be accumulated. However, since magnetic fields are three dimensional, a full magnetohydrodynamic (MHD) code should be applied to fully interpret the convective flows and the dynamics of the processes of emerging and cancelling magnetic features.

\section{Acknowledgements}

This work was supported by the European Commission through the SOLAIRE Network (MTRN-CT-2006-035484).

\section{References}

Berger, M. A. \& Field, G. B. 1984, J. Fluid Mech., 147, 133

Chae, J. 2001, ApJ, 560, L95

Mackay, D. H., Priest, E. R., \& Lockwood, M. 2002, Sol. Phys., 209, 287

Mackay, D. H. \& Gaizauskas, V. 2003, Sol. Phys., 216, 121

Park, J., Chae, J., Haimin, W. 2010, ApJ, in press

Parnell C. E. 2001, Sol. Phys, 200, 23

Priest, E. R., Heyvaerts, J. F., \& Title, A. M. 2002, ApJ, 576, 533

Romano P., Contarino L., \& Zuccarello F. 2003, Sol.Phys., 218, 137

Smyrli A., Zuccarello, F., Romano, P., Zuccarello, F. P., Guglielmino, S. L., Spadaro, D., Hood, A. W., \& Mackay, D. H., 2010, $A \& \mathcal{E} A$, in press 